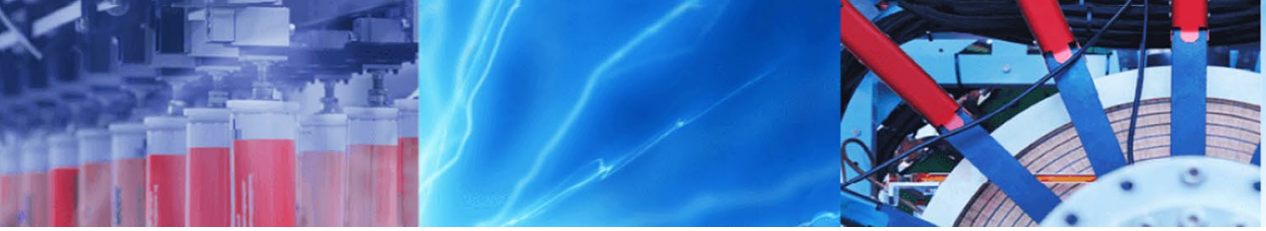

Research Article

\title{
Effects of Dufour and thermal diffusion on unsteady MHD free convection and mass transfer flow through an infinite vertical permeable sheet
}

\author{
Md. Hasanuzzaman ${ }^{1} \mathbb{D} \cdot$ Md. Abul Kalam Azad ${ }^{1} \cdot$ Md. Mosharrof Hossain $^{2}$
}

Received: 2 June 2021 / Accepted: 26 October 2021

Published online: 22 November 2021

(c) The Author(s) $2021 \quad$ OPEN

\begin{abstract}
In this paper, the effects of Dufour and thermal diffusion and on unsteady MHD (magnetohydrodynamic) free convection and mass transfer flow through an infinite vertical permeable sheet have been investigated numerically. The nondimensional governing equations are solved numerically by using the superposition method with the help of "Tec plot" software. The numerical solution regarding the non-dimensional velocity, temperature, and concentration variables against the non-dimensional coordinate variable has been carried out for various values of pertinent numbers and parameters like the suction parameter $\left(v_{0}\right)$, Prandtl number $\left(P_{r}\right)$, magnetic parameter $(M)$, Dufour number $\left(D_{f}\right)$, Soret number $\left(S_{0}\right)$, Schmidt number $\left(S_{c}\right)$, and for constant values of modified local Grashof number $\left(G_{m}\right)$ and local Grashof number $\left(G_{r}\right)$ .The velocity field decreases for increasing the suction parameter which is focusing on the common fact that the usual suction parameter stabilizing the effect on the boundary layer growth. The thermal boundary layer thickness becomes thinner for rising values of the Dufour and Soret numbers. The skin friction enhances for uplifting values of Soret number and Dufour number but reduces for moving suction parameter, Magnetic force number, Prandtl number, and Schmidt number. The heat transfer rate increases for increasing the suction parameter, Dufour number, Prandtl number, and Soret number. The mass transfer rate increases for enhancing the values of suction parameter, Magnetic force number, Soret number, and Prandtl number but decreases for Dufour number and Schmidt number.
\end{abstract}

Keywords MHD $\cdot$ Heat and mass transport $\cdot$ Dufour effect $\cdot$ Soret effect $\cdot$ Permeability

$\begin{array}{llll}\text { List of symbols } & k & \text { Thermal conductivity } \\ u & \text { Velocity component along the } x \text {-axis, } \mathrm{ms}^{-1} & C_{\mathrm{p}} & \text { Specific heat at constant pressure } \\ \mathrm{MHD} & \text { Magnetohydrodynamic } & k_{\mathrm{T}} & \text { Thermal diffusion ratio } \\ \boldsymbol{J} & \text { Density of current } & \sigma & \text { Similarity parameter } \\ T_{\mathrm{w}} & \text { Wall temperature, } \mathrm{k}^{-1} & G_{r} & \text { Local Grashof number } \\ \mathrm{C} & \text { Fluid concentration, } \mathrm{kg} \mathrm{m}^{-3} & M & \text { Magnetic parameter } \\ C_{\infty} & \text { Free stream concentration } & D_{f} & \text { Dufour number } \\ U_{0}(t) & \text { Uniform surface velocity } & S_{c} & \text { Schmidt number } \\ g & \text { Acceleration due to gravity, } \mathrm{ms}^{-2} & \tau & \text { Local skin friction coefficient } \\ \beta & \text { Volumetric expansion coefficient with } & S_{h} & \text { Sherwood number } \\ & \text { temperature } & \theta & \text { Dimensionless temperature }\end{array}$

Md. Hasanuzzaman, hasanuzzaman@math.kuet.ac.bd| 'Department of Mathematics, Khulna University of Engineering and Technology, Khulna 9203, Bangladesh. ${ }^{2}$ Department of Mathematics, Government BM College, Barishal 8200, Bangladesh.

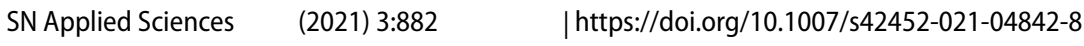


$\checkmark \quad$ Velocity component along the $y$-axis, $\mathrm{ms}^{-1}$

B Uniform magnetic field, $\mathrm{Am}^{-1}$

$T \quad$ Temperature of fluid, $\mathrm{k}^{-1}$

$T_{\infty} \quad$ Free stream temperature

$C_{\mathrm{w}} \quad$ Wall concentration, $\mathrm{kg} \mathrm{m}^{-3}$

$\rho \quad$ Fluid density, $\mathrm{kg} \mathrm{m}^{-3}$

$v(t) \quad$ Suction velocity

$v \quad$ Kinematic viscosity, $\mathrm{m}^{-2} \mathrm{~s}^{-1}$

$\beta^{*} \quad$ Volumetric expansion coefficient with

concentration

$C_{s} \quad$ Concentration susceptibility

$T_{\mathrm{m}} \quad$ Fluid mean temperature

$D_{\mathrm{m}} \quad$ Mass diffusivity coefficient

$v_{0} \quad$ Suction and blowing

$G_{\mathrm{m}} \quad$ Modified local Grashof number

$P_{r} \quad$ Prandtl number

$S_{0} \quad$ Soret number

$t \quad$ Time, $\mathrm{s}$

$N_{u} \quad$ Nusselt number

$f^{\prime} \quad$ Dimensionless velocity

$\phi \quad$ Dimensionless concentration

\section{Introduction}

Magnetohydrodynamic (MHD) fluid flow through a porous medium has many significant roles in pure science, engineering, technological, and biomedical fields such as MHD power generators, MHD accelerators, blood flow measurements, electrolytes, ionized gases, traveling waves tubes, metal-working processes, propulsion units, and control fusion research. A porous medium is a substance that has pores. Numerous natural substances such as rocks, soil, wood, cork can be considered as porous media. The porous media is used in various science, engineering, and biomedical applications such as soil and rock mechanics, petroleum technology, the dying process, material science, human lungs, and small bold capillaries. Porous medium describes the two prompt properties, namely porosity and permeability. Porosity evaluates the amount of fluid tackled by the material, while permeability measures quantitatively the ability of the porous medium to permit fluid flow. In boundary layer flow problems, MHD controls the force and heat exchanged by the surface. Srinivas and Muthuraj [1] debilitated the homotopy analysis method to find an approximate solution for the MHD flow of viscous incompressible fluid with thermal radiation and porosity effects. Raftari and Vajravelu [2] investigated the magnetohydrodynamic viscoelastic fluid characteristic through a wall. Heat transfer for micropolar fluid embedded in a permeable medium was explored by Xinhui et al. [3]. Kothandapani and Prakash [4] have discussed the influence of chemical reactions, inclined magnetic fields, and thermal

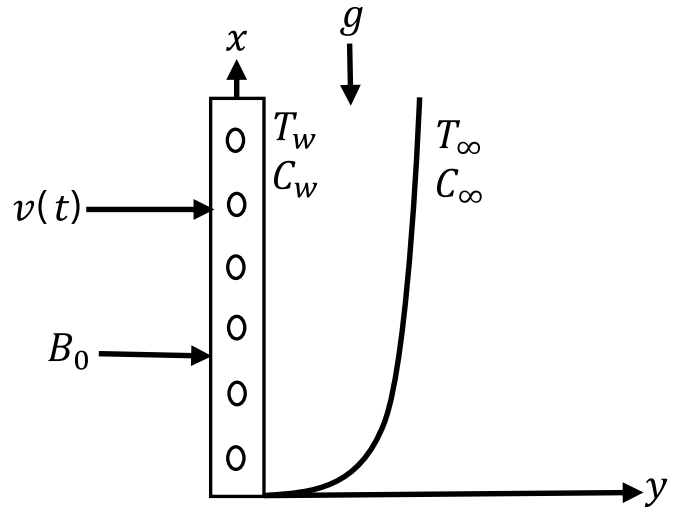

Fig. 1 Physical model and coordinate system

radiation over a vertical channel by using the method of homotopy perturbation. MHD flow over an exponentially stretching surface embedded by permeable medium with thermal radiation effect was elucidated by Sharma and Gupta [5]. Kumar et al. [6] examined the influences of a constant heat source on fractional exothermic reactions model with exponential, power, and Mittag-Leffler laws in a permeable medium. The boundary layer flow for viscous fluid over a flat sheet was evaluated by Sushila et al. [7].

In recent years, the analysis of Dufour and Soret effects has had great significance and attracted various scientific researchers due to its applications in engineering, industry, and geosciences such as hydrology, petrology, turbine blades, foam combustion, and gas-particle trajectories. The heat transfer and mass transfers caused by the concentration and the temperature gradient are said the Dufour effect and Soret effect, respectively. Soret and Dufour effects on steady MHD free convection and mass transfer flow through a semi-infinite vertical permeable sheet embedded in a permeable medium have been discussed by Alam and Rahman [8]. The transversely applied magnetic field effect on unsteady free convection and mass transport through an impulsively started infinite vertical permeable flat sheet in a permeable medium with Dufour and Soret effects has been explained by Alam and Rahman [9] (Fig. 1). The same problem in the case of MHD flow has been explained by Kafoussias and Williams [10]. They have completed their analytical studies by applying the Laplace transform method. The effect of thermal radiation and viscous dissipation on the two-dimensional MHD flow of viscoelastic Jeffrey fluid through the impermeable surface with heat generation/absorption effect has been discussed by Sharma and Gupta [11]. The effect of the chemical reaction and thermal radiation on threedimensional MHD viscous and incompressible flow in the presence of Dufour and Soret effects has been investigated by Sharma and Bhaskar [12]. The two-dimensional 
unsteady squeezing Casson fluid flow between permeable channels along with viscous dissipation, cross-diffusion, chemical reaction, and radiation effects has been analyzed by Bhaskar and Sharma $[13,14]$. Bhaskar and Sharma [13, 14] have investigated the effect of radiation and chemical reaction on the electrically conducting laminar flow incompressible mixed convective couple stress fluid, saturated past an upstanding passage in a permeable medium with Hall current, Joule heating, Dufour, and Soret effects. Nabil and Mahmoud [15] studied the effects of Dufour and Soret on the viscous fluid with mass and heat transfer flow past permeable medium on a shrinking plate. The effect of magnetic field and chemical reaction on unsteady free convection fluid flow through an infinite vertical permeable plate with thermal diffusion and diffusion-thermo effects have been also studied by Srinivasa Raju [16]. Further, the numerical results for the effects of thermal diffusion and diffusion-thermo on an unsteady free convection flow through an infinite vertical sheet with transverse magnetic field with thermal radiation and heat source have been observed by Srinivasa Raju et al. [16]. Saeed et al. [17] have examined the effect of heat generation/absorption and Joule heating on the transmission of thermal energy over a stretching cylinder theoretically. Also, they have investigated the entropy generation rate for spinning flow systems. Arshad Khan et al. [18, 19] have discussed the thermal analysis for bioconvection of hybrid nanofluid flowing upon a thin horizontally movable needle. They have also studied the influence of Brownian motion and thermophoretic forces on the flow system of the famous Buongiorno's model. More recently, using the hybrid nanoparticles containing silver and copper oxide, the efficiency to conduct the distribution of heat transmission for fluid flow happens upon a thin-shaped hot needle with water as the base fluid has been studied by Arshad Khan et al. [18, 19]. The effect of thermal radiation on the multi slip conditions on the magnetohydrodynamic (MHD) mixed convection unsteady flow of micropolar nanofluids upon a shrinking/stretching plate with a heat source has been investigated by Abdal et al. [20]. Hussain et al. [21] have investigated the thermal radiation effect on the boundary layer flow and heat transfer aspects of a nanofluid on a permeable plate. Habib et al. [22] have considered a mathematical analysis for slip effects on MHD nanofluid in the presence of gyrotactic microorganisms and electromagnetic fields with thermal radiation and activation energy. The double-diffusion effects on free convective flow over a vertical stretching surface embedded in a permeable medium with radiation, Dufour and Soret effects, and a homogeneous first-order chemical reaction was explained by Abdelraheem et al. [23]. A numerical model for the effects of Soret number, Dufour number, and variable viscosity on MHD mixed convection and mass transfer flow of an exponentially stretching vertical surface embedded in a permeable medium was developed by Ahmed and Sibanda [24].

The main outcome of this present study is to consider the above problems through an infinite vertical permeable sheet keeping into account the effects of Dufour and thermal diffusion. The novelty of this work is increased further by considering the thermal radiation and chemical reaction with the Runge-Kutta Merson integration scheme which has not been discussed yet. Computations have been performed for the vast range of the nondimensional numbers/parameters like suction parameter, magnetic number, Dufour number, Prandtl number, Soret number, and Schmidt number are discussed graphically. In addition to the skin friction coefficient, heat and mass transfer properties have been discussed with the tabular representations.

\section{Governing equations}

Let us consider an unsteady MHD free convection and mass transfer flow of the fluid of an electrically conducting viscous through an infinite vertical permeable sheet at $y=0$. We consider, the $x$-axis is along with the sheet in an upward direction and the plane of the sheet in the fluid is normal to the $y$-axis. Along the $y$-axis, the permeable sheet is assumed to be electrically non-conducting when a uniform magnetic field $\boldsymbol{B}$ is imposedon the sheet. The induced magnetic field is negligible for considering small enough flow magnetic Reynolds number compare with one of the researches [25]. Then the magnetic force lines are adjusted comparative to the fluid and $\boldsymbol{B}=\left(0, B_{0}, 0\right)$. The charge conservation equation $\nabla \cdot \boldsymbol{J}=0$ which gives us $J_{y}=$ constant, where the density of current is $\boldsymbol{J}=\left(J_{x}, J_{y}, J_{z}\right)$.The direction of propagation is considered only along the $y$-axis and does not have any variation along the $y$-axis and the derivative of $J_{y}$ with respect to $y$, namely $\frac{\partial J_{y}}{\partial y}=0$. Since the sheet is electrically non-conducting, this constant is zero and hence $J_{y}=0$. The fluid is assumed to have constant properties except that the influence of the density variations with temperature and concentration, which are considered only in the body force term. Under the above assumptions, the physical variables are functions of $y$ and $t$ only.

The one-dimensional problem under the above assumptions and Boussinesq approximation can be put in the following form [Alam and Rahman [9]]:

The Continuity Equation:

$$
\frac{\partial v}{\partial y}=0
$$


The Momentum Equation:

$\frac{\partial u}{\partial t}+v \frac{\partial u}{\partial y}=v \frac{\partial^{2} u}{\partial y^{2}}+g \beta\left(T-T_{\infty}\right)+g \beta^{*}\left(C-C_{\infty}\right)-\frac{\sigma^{\prime} B_{0}^{2} u}{\rho}$

The Energy Equation:

$\frac{\partial T}{\partial t}+v \frac{\partial T}{\partial y}=\frac{k}{\rho C_{p}} \frac{\partial^{2} T}{\partial y^{2}}+\frac{D_{\mathrm{m}} k_{\mathrm{T}}}{C_{\mathrm{s}} C_{\mathrm{p}}} \frac{\partial^{2} C}{\partial y^{2}}$

The Concentration Equation:

$\frac{\partial C}{\partial t}+v \frac{\partial C}{\partial y}=D_{m} \frac{\partial^{2} C}{\partial y^{2}}+\frac{D_{m} k_{\mathrm{T}}}{T_{\mathrm{m}}} \frac{\partial^{2} T}{\partial y^{2}}$

The associate boundary conditions for the present problem are

$t>0, u=U_{0}(t), v=v(t), T=T_{w}, C=C_{w}$ aty $=0$

$t>0, u=0, v=0, T \rightarrow T_{\infty}, C \rightarrow C_{\infty}$ aty $\rightarrow \infty$

where $u$ and $v$ are the velocity components in the $x-$ axis and $y-$ axis, respectively. $\rho$ is the fluid density, due to gravity $g$ is the acceleration, $v$ is the kinematic viscosity, $\beta^{*}$ is the expansion volumetric coefficient with concentration, the volumetric expansion coefficient with temperature is $\beta, T$ is the fluid temperature, $T_{\mathrm{w}}$ is the wall temperature and $T_{\infty}$ is the fluid temperature in the free stream, $C$ is the fluid concentration, $C_{w}$ is the wall concentration and $C_{\infty}$ is the fluid concentration in the free stream, the thermal conductivity of the sheet is $k, C_{s}$ is the concentration susceptibility, at constant pressure $C_{\mathrm{p}}$ is the specific heat, $T_{\mathrm{m}}$ is the mean temperature of the fluid, the thermal diffusion ratio is $k_{\mathrm{T}}$, and $D_{\mathrm{m}}$ is the mass diffusivity coefficient.

Introducing a similarity parameter $\sigma$ as

$\sigma=\sigma(t)$

where $\sigma$ is the length scale with time-dependent. The solution of Eq. (1) is considered in terms of this length scale as follows:

$v=v_{0} \frac{v}{\sigma}$

here at the sheet, the dimensionless normal velocity is $v_{0}$. If $v_{0}>0$ represents suction and $v_{0}<0$ represents blowing.

Upon introducing the following similarity variables

$\eta=\frac{y}{\sigma}, f(\eta)=\frac{u}{U_{0}}, \theta(\eta)=\frac{T-T_{\infty}}{T_{w}-T_{\infty}}, \phi(\eta)=\frac{C-C_{\infty}}{C_{w}-C_{\infty}}$

Applying Eqs. (7-9), Eqs. (1-4) are transformed into the non-dimensional coupled ordinary differential equations as follows: $f^{\prime \prime}+2 \xi f^{\prime}+G_{r} \theta+G_{m} \phi-M f=0$

$\theta^{\prime \prime}+2 \xi P_{r} \theta^{\prime}+P_{r} D_{f} \phi^{\prime \prime}=0$

$\phi^{\prime \prime}+2 \xi S_{c} \phi^{\prime}+S_{c} S_{0} \theta^{\prime \prime}=0$

The transformed boundary conditions are as follows:

$f=1, \theta=1, \phi=1$ at $\eta=0$

$f=0, \theta=0, \phi=0$ at $\eta \rightarrow \infty$

where $G_{r}=\frac{g \beta\left(T_{\mathrm{w}}-T_{\infty}\right) \sigma^{2}}{U_{0} v}$ is the local Grashof number, the modified local Grashof number is $G_{\mathrm{m}}=\frac{g \beta^{*}\left(C_{\mathrm{w}}-C_{\infty}\right) \sigma^{2}}{U_{0} v}$, Magnetic parameter is $M=\frac{\sigma^{\prime} B_{0}^{2} \sigma^{2}}{\rho v}$, Prandtl number is $P_{r}=\frac{\rho v C_{\mathrm{p}}}{k}$, Dufour number is $D_{f}=\frac{\rho D_{\mathrm{m}} k_{\mathrm{T}}\left(C_{\mathrm{w}}-C_{\infty}\right)}{C_{\mathrm{s}} C_{\mathrm{p}} v\left(T_{\mathrm{w}}-T_{\infty}\right)}$, Soret number is $S_{0}=\frac{D_{\mathrm{m}} k_{\mathrm{T}}\left(T_{\mathrm{w}}-T_{\infty}\right)}{v T_{\mathrm{m}}\left(C_{\mathrm{w}}-C_{\infty}\right)}$, Schmidt number is $S_{c}=\frac{v}{D_{\mathrm{m}}}$ and $\xi=\eta+\frac{v_{0}}{2}$

The flow parameters are the Nusselt number, the Sherwood number, and local Skin friction coefficient as follows:

$N_{u} \propto-\theta^{\prime}, S_{h} \propto-\phi^{\prime}, \tau \propto f^{\prime}$

\section{Numerical solution}

The solutions of Eqs. $(10,11$, and 12) with the boundary conditions (13-14) are obtained by using the superposition method ( $\mathrm{Na}$ 1979). The boundary value problem reduces to an initial value problem by using this superposition method that can easily be integrated out by an initial value solver. Thus, to reduce Eqs. $(10,11$, and 12$)$ to an initial value problem the function $(\eta), \theta(\eta)$ and $\phi(\eta)$ are, respectively, decomposed to

$f(\eta)=f_{1}(\eta)+\mu f_{2}(\eta)+\lambda f_{3}(\eta)+\delta f_{4}(\eta)$

$\theta(\eta)=\theta_{1}(\eta)+\mu \theta_{2}(\eta)+\lambda \theta_{3}(\eta)+\delta \theta_{4}(\eta)$

$\phi(\eta)=\phi_{1}(\eta)+\mu \phi_{2}(\eta)+\lambda \phi_{3}(\eta)+\delta \phi_{4}(\eta)$

where $\mu, \lambda$, and $\delta$ are constants, the physical significance of which is obtained later. Now substituting Eqs. (16-18) in Eqs. (10-12) and then equating the different coefficients to zero we obtain the following differential equations.

$f_{1}^{\prime \prime}+2 \xi f_{1}^{\prime}-M f_{1}+G_{r} \theta_{1}+G_{m} \phi_{1}=0$

$f_{2}^{\prime \prime}+2 \xi f_{2}^{\prime}-M f_{2}+G_{r} \theta_{2}+G_{m} \phi_{2}=0$ 


$$
\begin{aligned}
& f_{3}^{\prime \prime}+2 \xi f_{3}^{\prime}-M f_{3}+G_{r} \theta_{3}+G_{m} \phi_{3}=0 \\
& f_{4}^{\prime \prime}+2 \xi f_{4}^{\prime}-M f_{4}+G_{r} \theta_{4}+G_{m} \phi_{4}=0 \\
& \theta_{1}^{\prime \prime}+2 \xi P_{r} \theta_{1}^{\prime}+D_{f} P_{r} \phi_{1}^{\prime \prime}=0 \\
& \theta_{2}^{\prime \prime}+2 \xi P_{r} \theta_{2}^{\prime}+D_{f} P_{r} \phi_{2}^{\prime \prime}=0 \\
& \theta_{3}^{\prime \prime}+2 \xi P_{r} \theta_{3}^{\prime}+D_{f} P_{r} \phi_{3}^{\prime \prime}=0 \\
& \theta_{4}^{\prime \prime}+2 \xi P_{r} \theta_{4}^{\prime}+D_{f} P_{r} \phi_{4}^{\prime \prime}=0 \\
& \phi_{1}^{\prime \prime}+2 \xi S_{c} \phi_{1}^{\prime}+S_{c} S_{0} \theta_{1}^{\prime \prime}=0 \\
& \phi_{2}^{\prime \prime}+2 \xi S_{c} \phi_{2}^{\prime}+S_{c} S_{0} \theta_{2}^{\prime \prime}=0 \\
& \phi_{3}^{\prime \prime}+2 \xi S_{c} \phi_{3}^{\prime}+S_{c} S_{0} \theta_{3}^{\prime \prime}=0 \\
& S_{c} \phi_{4}^{\prime}+S_{c} S_{0} \theta_{4}^{\prime \prime}=0
\end{aligned}
$$

The initial values of the decomposed functions $f_{1}(\eta), f_{2}(\eta), f_{3}(\eta), f_{4}(\eta), \ldots$ etc. are now obtained through the boundary conditions (13-14) as

$$
\begin{aligned}
& f_{1}(\eta)=1.0, f_{2}(\eta)=0, f_{3}(\eta)=0, f_{4}(\eta)=0 \\
& \theta_{1}(\eta)=1.0, \theta_{2}(\eta)=0, \theta_{3}(\eta)=0, \theta_{4}(\eta)=0 \\
& \phi_{1}(\eta)=1.0, \phi_{2}(\eta)=0, \phi_{3}(\eta)=0, \phi_{4}(\eta)=0
\end{aligned}
$$

Again as $\eta \rightarrow \infty$, applying the boundary conditions (13, $14)$ in $(16,17$, and 18$)$ we get

$$
\mu=-\frac{f_{1}\left(\theta_{3} \phi_{4}-\theta_{4} \phi_{3}\right)+\theta_{1}\left(f_{4} \phi_{3}-f_{3} \phi_{4}\right)+\phi_{1}\left(f_{1} \theta_{4}-f_{4} \theta_{1}\right)}{f_{2}\left(\theta_{3} \phi_{4}-\theta_{4} \phi_{3}\right)+f_{1}\left(f_{4} \phi_{3}-f_{3} \phi_{4}\right)+\phi_{1}\left(f_{1} \theta_{4}-f_{4} \theta_{1}\right)}
$$

$$
\lambda=-\frac{f_{1}\left(\theta_{4} \phi_{2}-\theta_{2} \phi_{4}\right)+\theta_{1}\left(f_{2} \phi_{4}-f_{4} \phi_{2}\right)+\phi_{1}\left(\theta_{2} f_{4}-\theta_{4} f_{2}\right)}{f_{2}\left(\theta_{3} \phi_{4}-\theta_{4} \phi_{3}\right)+f_{1}\left(f_{4} \phi_{3}-f_{3} \phi_{4}\right)+\phi_{1}\left(f_{1} \theta_{4}-f_{4} \theta_{1}\right)}
$$

$\delta=-\frac{f_{1}\left(\theta_{2} \phi_{3}-\theta_{3} \phi_{2}\right)+\theta_{1}\left(f_{3} \phi_{2}-f_{2} \phi_{3}\right)+\phi_{1}\left(\theta_{3} f_{2}-\theta_{2} f_{3}\right)}{f_{2}\left(\theta_{3} \phi_{4}-\theta_{4} \phi_{3}\right)+f_{1}\left(f_{4} \phi_{3}-f_{3} \phi_{4}\right)+\phi_{1}\left(f_{1} \theta_{4}-f_{4} \theta_{1}\right)}$

In $(16,17$, and 18) all the functional values are obtained as

$$
\begin{aligned}
& \frac{\partial f(\eta)}{\partial \eta}=\frac{\partial f_{1}(\eta)}{\partial \eta}+\mu \frac{\partial f_{2}(\eta)}{\partial \eta}+\lambda \frac{\partial f_{3}(\eta)}{\partial \eta}+\delta \frac{\partial f_{4}(\eta)}{\partial \eta} \\
& \frac{\partial \theta(\eta)}{\partial \eta}=\frac{\partial \theta_{1}(\eta)}{\partial \eta}+\mu \frac{\partial \theta_{2}(\eta)}{\partial \eta}+\lambda \frac{\partial \theta_{3}(\eta)}{\partial \eta}+\delta \frac{\partial \theta_{4}(\eta)}{\partial \eta}
\end{aligned}
$$$$
\frac{\partial \phi(\eta)}{\partial \eta}=\frac{\partial \phi_{1}(\eta)}{\partial \eta}+\mu \frac{\partial \phi_{2}(\eta)}{\partial \eta}+\lambda \frac{\partial \phi_{3}(\eta)}{\partial \eta}+\delta \frac{\partial \phi_{4}(\eta)}{\partial \eta}
$$

Then by setting the missing slopes.

$$
\frac{\partial f(0)}{\partial \eta}, \frac{\partial \theta(0)}{\partial \eta} \text { and } \frac{\partial \phi(0)}{\partial \eta} \text { as } \frac{\partial f(0)}{\partial \eta}=\mu, \frac{\partial \theta(0)}{\partial \eta}=\lambda \text { and } \frac{\partial \phi(0)}{\partial \eta}=\delta \text {. }
$$

The initial conditions for the slopes of the decomposed functions are obtained easily. The well known Runge-Kutta Merson integration scheme has been used as an initial value solver to integrate the above mentioned Eqs. (10, 11 , and 12) and to obtain converged solutions which are presented graphically in Figs. 2, 3, and 4. The local values of the skin friction $(\tau)$, Nusselt number $\left(N_{u}\right)$, and Sherwood number $\left(S_{h}\right)$ are proportional to $-\partial f(0) / \partial \eta,-\partial \theta(0) / \partial \eta$ and $-\partial \phi(0) / \partial \eta$, respectively. The numerical values of the skin friction, Nusselt number andSherwood number are sorted in Tables 1-3.

\section{Results and discussions}

An investigation for unsteady MHD free convection and mass transfer flow through an infinite vertical permeable sheet has been investigated to analyze the effect of Dufour and thermal diffusion. The resulting initial value problems involving the set of ordinary differential equations (ODE) $(10,11$, and 12) including the boundary conditions (13-14) are solved numerically by applying the superposition method through "Tec plot 9.6" software. The effects of suction parameter $\left(v_{0}\right)$, the Dufour number $\left(D_{f}\right)$, the magnetic parameter $(M)$, the Prandtl number $\left(P_{r}\right)$, the Soret num$\operatorname{ber}\left(S_{0}\right)$, the Schmidt number $\left(S_{c}\right)$, and for constant values of local Grashof number $\left(G_{r}\right)$ and modified local Grashof number $\left(G_{m}\right)$ on velocity, temperature, and concentration distributions are plotted in Figs. (2, 3, and 4).The values $0.71,1.0$, and 7.0 are considered for $P_{r}\left(0.71\right.$ for air at $20^{0}$ and $1.0,7.0$ for water at $17^{\circ}$ ). The values $0.22,0.60$, and 0.75 are also considered for $S_{c}(0.75$ for Oxygen, 0.60 for vapor water, and 0.22 for Hydrogen). The other parameter values are, however, chosen arbitrarily.

\subsection{Velocity variation}

Figure $2 a-f$ demonstrates the variation of fluid velocity for various values of the suction parameter $\left(v_{0}\right)$,the magnetic parameter $(M)$, the Dufour number $\left(D_{f}\right)$, the Soret number 

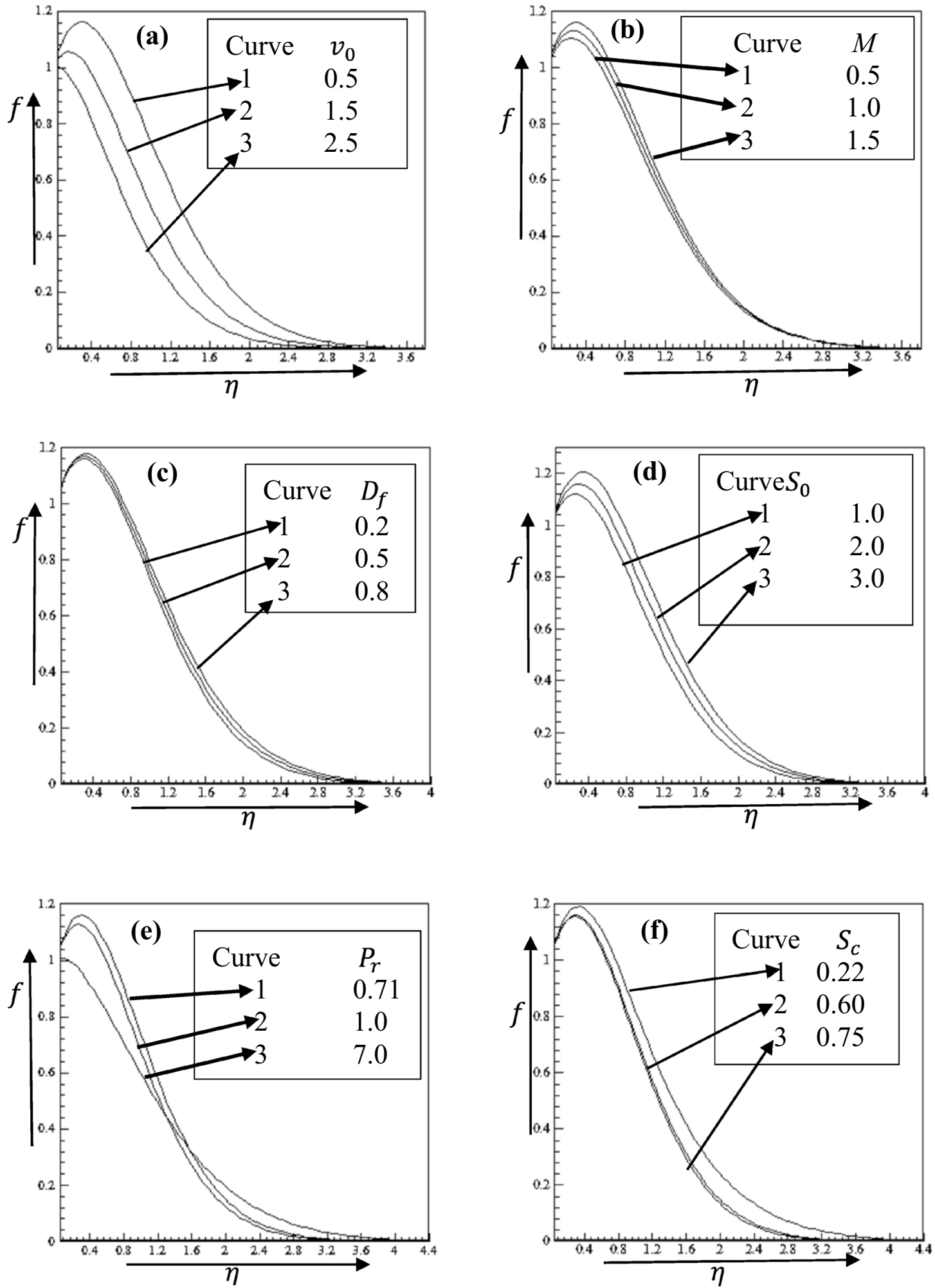

Fig. 2 Velocity profiles for the variation of $\mathbf{a} v_{0}, \mathbf{b} M, \mathbf{c} D_{f}, \mathbf{d} S_{0}$, e $P_{r}$, and $\mathbf{f} S_{c}$ 

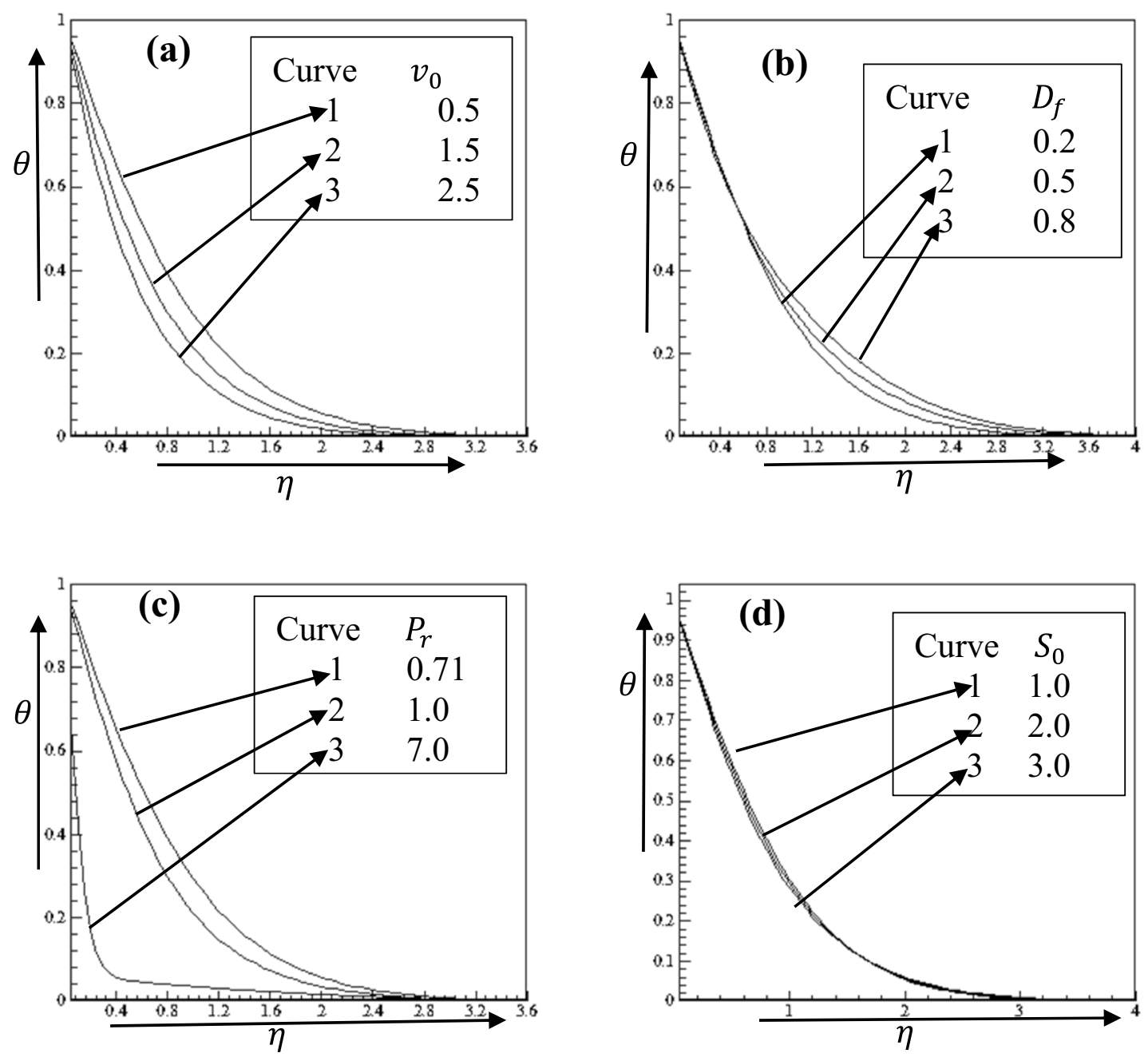

Fig. 3 Temperature profile for the variation of $\mathbf{a} v_{0}, \mathbf{b} D_{f}, \mathbf{c} P_{r}$, and $\mathbf{d} S_{0}$

$\left(S_{0}\right)$, the Prandtl number $\left(P_{r}\right)$ and the Schmidt number $\left(S_{c}\right)$ It can be concluded from Fig. $2 a$ that the velocity profiles decrease monotonically with the increase in the suction parameter $\left(v_{0}\right)$ indicating the usual fact that suction stabilizes the boundary layer growth. It is seen from Fig. $2 b$ that the velocity of the fluid reduces for uplifting values of magnetic parameter $(M)$. A resistive type of force like a drag force is produced for increasing the values of the magnetic parameter, which name is Lorentz force. The nature of Lorentz force interrupts the force on the velocity which reduces its motion. Therefore, the velocity decreases with the increase in magnetic field parameter $(M)$. Due to this physical phenomenon, the growing values for $M$ decline axial velocity of the fluid. It is found from Fig. $2 \mathrm{C}$ that an increase in the Dufour number $\left(D_{f}\right)$ causes a rise in the velocity throughout the boundary layer. However, a distinct velocity overshoot exists near the plate, and thereafter the profile falls to zero at the edge of the boundary layer. It is observed from Fig. $2 \mathrm{~d}$ that the thermal boundary layer thickness becomes thinner for rising values of the Soret number. So that the velocity increases with an increase in the Soret number. It is noticed from Fig. 2e that with the increase in Prandtl number $P_{r}$, the velocity profile decreases. Prandtl number is the ratio of momentum diffusivity to thermal diffusivity. An increase in $P_{r}$ the fluid becomes more viscous and for a smaller value of $P_{r}$ the momentum boundary layer thickness is increased which slows down the velocity of the fluid. With a rise in the Schmidt number $\left(S_{c}\right)$, the velocity profile reduction is shown in Fig. 2f. Physically, the Schmidt number represents the relative thickness of the momentum boundary layer and mass concentration boundary layer. Therefore, as $S_{c}$ increase, the momentum boundary layer is increased due to an increase in the kinematic viscosity of the fluid, and consequently, the fluid velocity decreases significantly. 

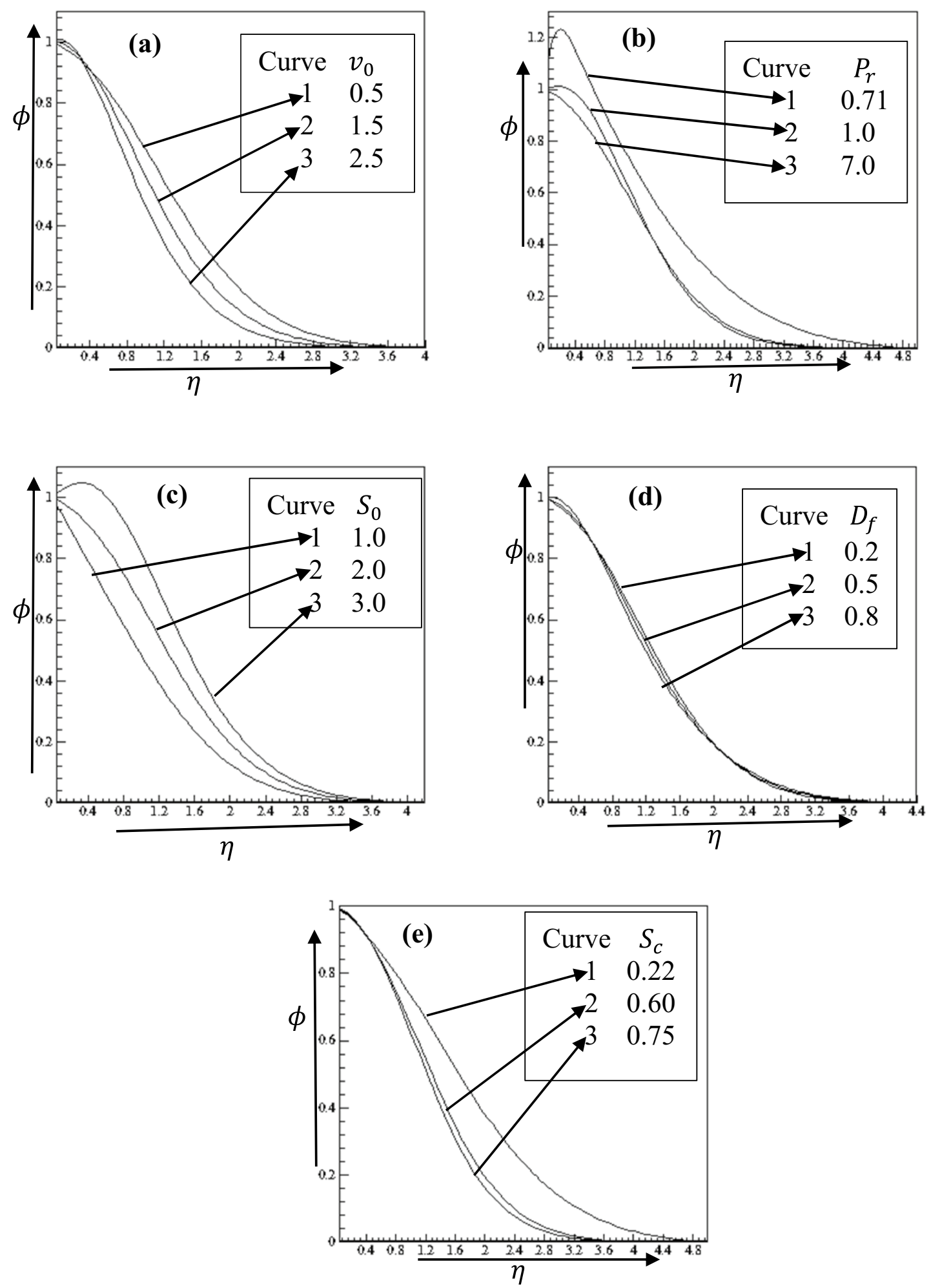

Fig. 4 Concentration profiles for the variation of $\mathbf{a} v_{0}, \mathbf{b} P_{r} \mathbf{c} S_{0}, \mathbf{d} D_{f}$, and e $S_{c}$

\subsection{Temperature variation}

The temperature profile for varying values of the suction parameter $\left(v_{0}\right)$, Dufour number $\left(D_{f}\right)$, Prandtl number $\left(P_{r}\right)$, and Soret number $\left(S_{0}\right)$ is displayed in Fig. 3a-d. It is noticed from Fig. $3 a$ that the thickness of the thermal boundary layer reduces for uplifting values of the suction parameter 
Table 1 Computed values for the local skin friction, rate of heat transfer, and rate of mass transfer for different values of $v_{0}$ and $D_{f}$ taking $G_{r}=10.0, G_{m}=5.0, M=0.5, S_{0}=0.5, P_{r}=0.71$ and $S_{c}=0.6$ as fixed

\begin{tabular}{lllll}
\hline$v_{0}$ & $D_{f}$ & $\tau$ & $-\theta^{\prime}$ & \multicolumn{1}{l}{$-\phi^{\prime}$} \\
\hline 0.5 & 0.2 & 1.784958492 & 1.419837320 & 0.22187363 \\
1.5 & 0.2 & 1.107044722 & 1.973658468 & 0.39512490 \\
2.5 & 0.2 & 0.252584350 & 2.580813500 & 0.39738800 \\
0.5 & 0.5 & 1.828867473 & 1.482323460 & 0.13702065 \\
0.5 & 0.8 & 1.871626340 & 1.657527020 & -0.82259632 \\
\hline
\end{tabular}

$\left(v_{0}\right)$. As a result, the temperature decreases for rising the suction parameter. From Fig. 3 (b), it is observed that the thermal boundary layer thickness and the temperature distribution for uplifting the values Dufour number $\left(D_{f}\right)$. Energy transfer takes place at a higher rate and, therefore, the temperature increases. It has been seen from Fig. $3 \mathrm{c}$ that the temperature decreases for enhancing the Prandtl number $\left(P_{r}\right)$. When the Prandtl number increases, then the thermal diffusivity of fluid particles reduces, and hence its temperature reduces. It is noticed from Fig. $3 d$ that the temperature profile reduces with a rise in Soret number $\left(S_{0}\right)$. With an increase in the values of Soret number the thermal boundary layer thickness decrease, and, thus the temperature reduces.

\subsection{Concentration variation}

The effects of suction parameter $\left(v_{0}\right)$, Prandtl number $\left(P_{r}\right)$, Soret number $\left(S_{0}\right)$, Dufour number $\left(D_{f}\right)$, and Schmidt number $\left(S_{c}\right)$ on the concentration profiles are plotted in Figs. 4a-e. The concentration decreases for rising the values of the suction parameter $\left(v_{0}\right)$ is shown in Fig. 4a. Sucking decelerated fluid particles through the permeable wall reduces the growth of the fluid boundary layer as well as thermal and concentration boundary layers. It is seen from Fig. $4 \mathrm{~b}$ that the larger values of Prandtl number $\left(P_{r}\right)$ causes a reduction in the fluid particle's concentration. Therefore, the nanoparticles concentration profile decreases with increases in $P_{r}$. It is observed from Fig. 4c that the concentration of the fluid increases for growing values of Soret number. In the Soret phenomenon, temperature gradient affected the concentration distribution. So, higher values of the Soret number result in higher convective flow and, hence, concentration increases. It is evident from Fig. $4 \mathrm{~d}$ that concentration decreases with a rise in Dufour number. The temperature difference between the fluid and the wall decreases with an increment in Dufour number, causing more heat to be transferred to the fluid which affects the fluid's viscosity. Hence, the concentration profile decreases. It is determined from Fig. 4e that the concentration of fluid reduces for enhancing values of Schmidt number $\left(S_{c}\right)$. In fact, when $S_{c}$ increases then molecular/ mass diffusivity of the fluid reduces that ultimately reduces the concentration of fluid particles.

\subsection{Skin friction, heat and concentration rate}

The various values of skin friction, rate of heat transfer, and rate of nanoparticle concentration have been presented in Tables 1, 2, and 3 for different values of mentioned parameters. From Table 1, it is observed that the skin friction reduces for moving suction parameter, Magnetic force number, Prandtl number, and Schmidt number but increases for uplifting values of Soret number and Dufour number. The heat transfer rate increases for increasing the suction parameter, Dufour number, Prandtl number, and Soret number is shown in Table 2. It is seen from Table 3 that the mass transfer rate increases for enhancing the values of the suction parameter, Magnetic force number, Soret number, and Prandtl number but decreases for Dufour number and Schmidt number.

Table 2 Computed values for the local skin friction, rate of heat transfer, and rate of mass transfer for different values of $M$ and $S_{0}$ taking $G_{r}=10.0, G_{m}=5.0, v_{0}=0.5, D_{f}=0.5, P_{r}=0.71$ and $S_{c}=0.6$ as fixed

\begin{tabular}{lllll}
\hline$M$ & $S_{0}$ & $\tau$ & $-\theta^{\prime}$ & $-\phi^{\prime}$ \\
\hline 0.5 & 1.0 & 1.784958492 & 1.4198373200 & 0.22187363 \\
1.0 & 1.0 & 1.572174200 & 1.4198413397 & 0.22188526 \\
1.5 & 1.0 & 1.371121700 & 1.4198464360 & 0.22190479 \\
0.5 & 2.0 & 1.555121620 & 1.3539478400 & 0.78080973 \\
0.5 & 3.0 & 2.028048486 & 1.4981375278 & 0.83033981 \\
\hline
\end{tabular}

Table 3 Computed values for the local skin friction, rate of heat transfer, and rate of mass transfer for different values of $P_{r}$ and $S_{c}$ taking $G_{r}=10.0, G_{\mathrm{m}}=5.0, v_{0}=0.5, D_{f}=0.5, M=0.5$ and $S_{0}=2.0$ as fixed

\begin{tabular}{lllll}
\hline$P_{r}$ & $S_{c}$ & $\tau$ & $-\theta^{\prime}$ & $-\phi^{\prime}$ \\
\hline 0.71 & 0.22 & 1.934421900 & 1.403163282 & 0.244711110 \\
1.0 & 0.22 & 1.590006500 & 1.822974568 & 0.289813788 \\
7.0 & 0.22 & 0.386611000 & 2.076471560 & 4.3426240450 \\
0.71 & 0.60 & 1.784958492 & 1.419837320 & 0.2218736300 \\
0.71 & 0.75 & 1.758096290 & 1.428434320 & 0.1710122012 \\
\hline
\end{tabular}




\section{Conclusions}

A numerical investigation of unsteady MHD free convection and mass transfer flow through an infinite vertical permeable sheet has been performed under the Dufour and thermal diffusion effects. From the numerical simulations, the following conclusions may be drawn:

- The skin friction coefficient increases about $5 \%$ and $14 \%$ due to increasing Dufour number $(0.2-0.8)$ and Soret number (1.0-3.0), respectively. On the other hand, increasing the suction parameter (0.5-1.5), Magnetic force number (0.5-1.0), Prandtl number (0.71$1.0)$, and Schmidt number (0.22-0.75) decreases the skin friction by $38 \%, 23 \%, 18 \%$ and $10 \%$, respectively.

- Rising values of the suction parameter (0.5-1.5), Dufour number (0.2-0.8), Prandtl number (0.71-1.0), and Soret number (1.0-3.0) increases the heat transfer rate approximately $39 \%, 17 \%, 30 \%$, and $6 \%$, respectively.

- Rate of mass transfer increases about $78 \%$ and $18 \%$ for suction parameter (0.5-1.5) and Prandtl number (0.71-1.0), respectively, but decreases about $38 \%$ and $30 \%$ for enhancing the Dufour number $(0.2-0.5)$ and Schmidt number (0.22-0.75), respectively.

The outcome of this present research may be useful for paper production, suspensions, and coating, the technology of heat exchangers, exploiting of materials processing, drying, water body evaporation at the surface, etc.

\section{Declarations}

Conflict of interest The authors declare that they have no known competing financial interests or personal relationships that could have appeared to influence the work reported in this paper.

Open Access This article is licensed under a Creative Commons Attribution 4.0 International License, which permits use, sharing, adaptation, distribution and reproduction in any medium or format, as long as you give appropriate credit to the original author(s) and the source, provide a link to the Creative Commons licence, and indicate if changes were made. The images or other third party material in this article are included in the article's Creative Commons licence, unless indicated otherwise in a credit line to the material. If material is not included in the article's Creative Commons licence and your intended use is not permitted by statutory regulation or exceeds the permitted use, you will need to obtain permission directly from the copyright holder. To view a copy of this licence, visit http://creativecommons. org/licenses/by/4.0/.

\section{References}

1. Srinivas S, Muthuraj R (2010) Effects of thermal radiation and space porosity on MHD mixed convection flow in a vertical channel using homotopy analysis method. Commun Nonlinear Sci 15(20):98-108

2. Raftari B, Vajravelu K (2012) Homotopy analysis method for MHD viscoelastic fluid flow and heat transfer in a channel with a stretching wall. Commun Nonlinear Sci 17(41):49-62

3. Xinhui S, Liancun Z, Ping L, Xinxin Z, Yan Z (2013) Flow and heat transfer of a micropolar fluid in a porous channel with expanding or contracting walls. Int J Heat Mass Transf 67(8):85-95

4. Kothandapani M, Prakash J (2015) Effects of thermal radiation and chemical reactions on peristaltic flow of a Newtonian nanofluid under inclined magnetic field in a generalized vertical channel using homotopy perturbation method. Asia-Pac J Chem Eng 10(2):59-72

5. Sharma K, Gupta S (2016) Analytical study of MHD boundary layer flow and heat transfer towards a porous exponentially stretching sheet in presence of thermal radiation. Int J Adv Appl Math Mech 4:1-10

6. Kumar D, Singh J, Tanwar K, Baleanu D (2019) A new fractional exothermic reactions model having constant heat source in porous media with power, exponential and Mittag-Leffler laws. Int J Heat Mass Transf 138(12):22-27

7. Sushila SJ, Shishodia YS (2013) An efficient analytical approach for MHD viscous flow over a stretching sheet via homotopy perturbation sumudu transform method. Ain Shams Eng J 4(5):49-55

8. Alam MS, Rahman MM (2005) Dufour and Soret effects on MHD free convective heat and mass transfer flow past a vertical flat plate embedded in a porous medium. J Naval Architect Mar Eng 2(1):55-65

9. Alam MS, Rahman MM (2006) Dufour and soret effects on mixed convection flow past a vertical porous flat plate with variable suction. Nonlinear Anal Model Control 11:3-12

10. Kafoussias NG, Williams EW (1995) Thermal-diffusion and diffusion-thermo effects on mixed free-forced convective and mass transfer boundary layer flow with temperature dependent viscosity. Int J Eng Sci 33:1369-1384

11. Sharma K, Gupta S (2017) Viscous dissipation and thermal radiation effects in MHD flow of Jeffrey nanofluid through impermeable surface with heat generation/absorption. Nonlinear Eng 6(2):153-166

12. Sharma K, Bhaskar K (2020) Influence of soret and Dufour on threedimensional MHD flow considering thermal radiation and chemical reaction. Int J Appl Comput Math 6(1):3

13. Bhaskar K, Sharma K (2021) Exploration of soret and Dufour effects on mixed convection couple stress fluid flow through an upstanding passage. Spec Topics Rev Porous Media 12(4):17-32

14. Bhaskar K, Sharma K (2021) Unsteady MHD squeezing viscous casson fluid flow in upright channel with cross-diffusion and thermal radiactive effects. Indian J Phys 95(7):1453-1467

15. Eldabe N, Zeid MA (2013) Thermal diffusion and diffusion thermo effects on the viscous fluid flow with heat and mass transfer through porous medium over a shrinking sheet. J Appl Math 2013:1-11

16. Raju RS (2016) Combined influence of thermal diffusion and diffusion thermo on unsteady hydromagnetic free convective fluid flow past an infinite vertical porous plate in presence of chemical reaction. J Inst Eng (India) Series C 97(4):505-515

17. Islam S, Khan A, PoomKumam HA, Shah Z, Khan W, Zubair M, Jawad M (2020) Radiative mixed convection flow of maxwell nanofluid over a stretching cylinder with joule heating and heat source/sink effects. Int Commun Heat Mass Transfer. https://doi. org/10.1016/j.icheatmasstransfer.2020.104979

18. Khan A, Saeed A, Tassaddiq A, Gul T, Kumam P, Ali I, Kumam W (2021) Bio-convective and chemically reactive hybrid nanofluid flow upon a thin stirring needle with viscous dissipation. Sci Rep 11(8066):1-17. https://doi.org/10.1038/s41598-021-86968-8 
19. Khan A, Kumam W, Khan I, Saeed A, Gul T, Kumam PID, Ali I (2021) Chemically reactive nanofluid flow past a thin moving needle with viscous dissipation, magnetic effects and hall current. PLoS ONE 16(4):e0249264. https://doi.org/10.1371/journal.pone.0249264

20. Abdal S, Hussain S, Siddique I, Ahmadian A, Ferrara M (2021) On solution existence of MHD Casson nanofuid transportation across an extending cylinder through porous media and evaluation of priori bounds. Sci Reports 11:7799

21. Hussain F, Abdal S, Abbas Z, Hussain N, Adnan M, Ali B, Zulqarnain RM, Ali L, Younas S (2020) Buoyancy effect on MHD slip flow and heat transfer of a nanofluid flow over a vertical porous plate. Sci Inq $\operatorname{Rev}(\mathrm{SIR}) 4(1): 1-16$

22. Habib D, Salamat N, Abdal S, Siddique I, Ang MC, Ahmadian A (2021) On the role of bioconvection and activation energy for time dependent nanofluid slip transpiration due to extending domain in the presence of electric and magnetic fields. Ain Shams Eng J (in press)
23. Abdelraheem MA, Mansour MA, Chamkha AJ (2011) Effects of soret and dufour numbers on free convection over isothermal and adiabatic stretching surfaces embedded in porous media. J Porous Media 14:67-72

24. Ahmed AK, Sibanda P (2014) Effect of temperature-dependent viscosity on MHD mixed convective flow from an exponentially stretching surface in porous media with cross-diffusion. Spec Top Rev Porous Media: Int J 5:157-170

25. Pai SI (1962) magnetogasdynamics and plasma dynamics. Springer Verlag, New York

Publisher's Note Springer Nature remains neutral with regard to jurisdictional claims in published maps and institutional affiliations. 